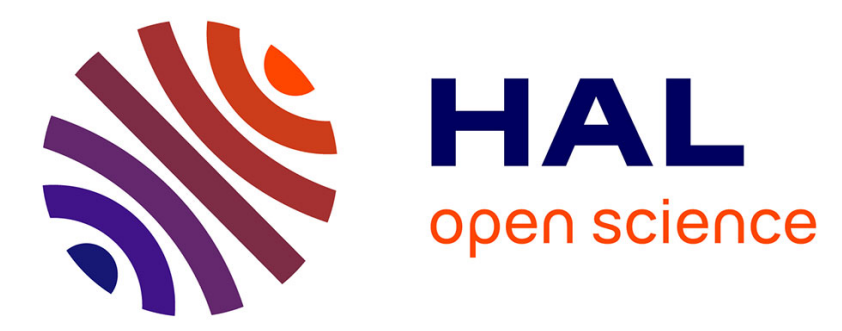

\title{
Femtoscopy within a hydrodynamic approach based on flux tube initial conditions
}

\author{
Klaus Werner, Iurii Karpenko, Tanguy Pierog, Marcus Bleicher, Kirill
} Mikhailov

\section{- To cite this version:}

Klaus Werner, Iurii Karpenko, Tanguy Pierog, Marcus Bleicher, Kirill Mikhailov. Femtoscopy within a hydrodynamic approach based on flux tube initial conditions. Physics of Particles and Nuclei Letters / Pis 'ma v Zhurnal Fizika Elementarnykh Chastits i Atomnogo Yadra, 2012, 9, pp.244-247. 10.1134/S1547477112030144 . in2p3-00689455

\section{HAL Id: in2p3-00689455 https://hal.in2p3.fr/in2p3-00689455}

Submitted on 23 Jun 2021

HAL is a multi-disciplinary open access archive for the deposit and dissemination of scientific research documents, whether they are published or not. The documents may come from teaching and research institutions in France or abroad, or from public or private research centers.
L'archive ouverte pluridisciplinaire HAL, est destinée au dépôt et à la diffusion de documents scientifiques de niveau recherche, publiés ou non, émanant des établissements d'enseignement et de recherche français ou étrangers, des laboratoires publics ou privés.

\section{(c)(1)}

Distributed under a Creative Commons Attribution| 4.0 International License 


\title{
Femtoscopy within a Hydrodynamic Approach Based on Flux Tube Initial Conditions ${ }^{1}$
}

\author{
K. Werner ${ }^{a}$, Iu. Karpenko ${ }^{a, b}$, T. Pierog ${ }^{c}$, M. Bleicher ${ }^{d}$, and K. Mikhailov ${ }^{e}$ \\ ${ }^{a} S U B A T E C H$, University of Nantes-IN2P3/CNRS-EMN, Nantes, France \\ ${ }^{b}$ Bogolyubov Institute for Theoretical Physics, Kiev \\ ${ }^{c}$ KIT, Institut für Kernphysik, Karlsruhe, Germany \\ ${ }^{d}$ FIAS, JW Goethe Universität, Frankfurt am Main, Germany \\ ${ }^{e}$ Institute for Theoretical and Experimental Physics, Moscow \\ e-mail:werner@subatech.in2p3.fr, karpenko@bitp.kiev.ua,pierog@ik.fzk.de, \\ bleicher@th.physik.uni-frankfurt.de, kmikhail@itep.ru
}

\begin{abstract}
In $p p$ scattering at LHC energies, large numbers of elementary scatterings will contribute significantly, and the corresponding high multiplicity events will be of particular interest. Elementary scatterings are parton ladders, identified with color flux tubes. In high multiplicity events, many of these flux tubes are produced in the same space region, creating high energy densities. We argue that there is good reason to employ the successful procedure used for heavy ion collisions: matter is assumed to thermalize quickly, so that the energy from the flux tubes can be taken as initial condition for a hydro-dynamic expansion. This scenario gets spectacular support from very recent results on Bose-Einstein correlations in $p p$ scattering at $900 \mathrm{GeV}$ at the LHC.
\end{abstract}

EPOS is a multiple scattering model in the spirit of the Gribov-Regge approach [1]. Here, one does not refer to simply multiple hard scatterings, the elementary processes correspond to complete parton ladders, which means hard scatterings plus initial state radiation. In this case, this elementary process carries an important fraction of the available energy, and therefore we treat very carefully the question of energy sharing in the multiple scattering process. Open and closed ladders have to be considered, in order to have a consistent quantum mechanical treatment. The corresponding graphs are squared, and we employ cutting rule techniques and Markov chains to obtain finally partial cross sections. The cut parton ladders are identified with longitudinal color fields or flux tubes, treated via relativistic string theory.

In case of very high energy $p p$ collisions (at the LHC) or heavy ion scatterings already at RHIC, many flux tubes overlap and produce high energy densities. Let us consider the energy density at an early time in an AuAu scattering at RHIC, as obtained from an EPOS simulation [1]. The energy density shows a very bumpy structure concerning the $x-y$ dependence (transverse coordinates), whereas the variation with the space-time rapidity $s$ is small. There are in particular peaks in the $x-y$ plane, which show up at the same position at different values of $\eta_{s}$. So we have sub-flux-

\footnotetext{
${ }^{1}$ The article is published in the original.
}

tubes which exhibit a long range structure in the longitudinal variable $\eta_{s}$, with a typical transverse width of the order of a fermi. This is exactly the width we obtain if we compute the initial energy density in protonproton scattering at the LHC. This means, if a hydrodynamic treatment is justified for $\mathrm{Au}-\mathrm{Au}$ collisions at RHIC, it is equally justified for $p p$ scattering at the LHC, provided the energy densities are high enough, which seems to be the case.

We are therefore going to employ a new tool for treating very high energy hadronic interactions including a hydrodynamic evolution (even in $p p$ ), as explained in [1]. In [1], we test the approach by investigating all soft observables of heavy ion physics, in case of AuAu scattering at $200 \mathrm{GeV}$. Let us consider proton-proton scattering now. In [2], we compare three different scenarios: the full calculations, including hydro evolution and hadronic cascade (full), the calculation without hadronic cascade (no casc), and the calculation without hydro and without cascade (base). Concerning particle spectra, we refer to [2], where we compare the corresponding calculations with experimental data, for $p p$ scattering at $900 \mathrm{GeV}$.

The space-time evolution of the "full" hydrodynamic approach will be completely different compared to the "base" approach, where particles are directly produced from breaking strings, as can be seen from Fig. 1, where we plot the distribution of formation points of $\pi^{+}$as a function of the radial distance $r=$ 


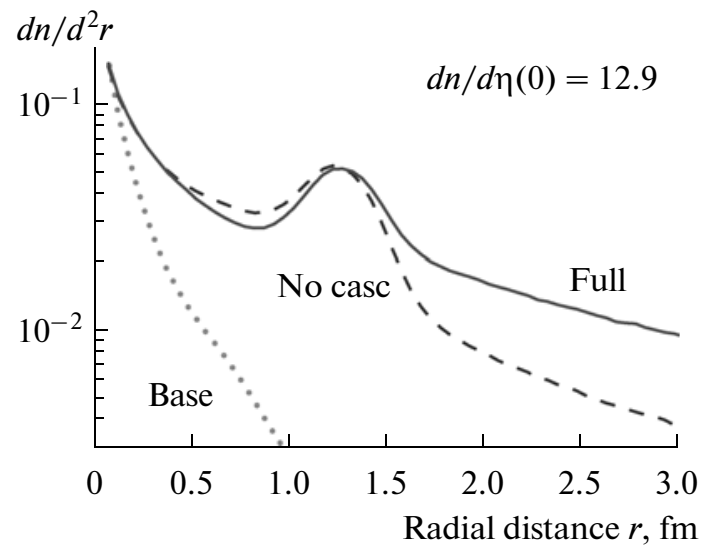

Fig. 1. (Color online) The distribution of formation points of $\pi+$ as a function of the radial distance in a high-multiplicity event from $p p$ scattering at $900 \mathrm{GeV}$, for the following scenarios: the full calculation (solid line), a calculation without hadronic cascade (dashed), and a calculation without hydro and without cascade (dotted).

$\sqrt{x^{2}+y^{2}}$ (in the $p p$ center-of-mass system (cms)) for the three scenarios. Only particles with space-time rapidities around zero are considered. All calculations in this paper refer to high-multiplicity events in $p p$ scattering at $900 \mathrm{GeV}$, with a mean $d n / d \eta(0)$ equal to 12.9. The "base calculation" (dotted line) gives as expected a steeply falling distribution as a function of $r$. In the two cases involving a hydrodynamic evolution, particle production is significantly delayed, even more in the case of the full calculation, with hadronic cascade. The bump in the two latter scenarios is due to particles being produced from the fluid, the small $p_{t}$ contribution is due to corona particles.

This particular space-time behavior of the hydrodynamic expansions should clearly affect Bose-Einstein correlations-what we are going to investigate in the following. There is a long history of so-called femtoscopic methods [4], where the study of two-particle correlations provides information about the source function $S\left(\mathbf{P}, r^{\prime}\right)$, being the probability of emitting a pair with total momentum $\mathbf{P}$ and relative distance $\mathbf{r}$ '. Under certain assumptions, the source function is related to the measurable two-particle correlation function $\mathrm{CF}(\mathbf{P}, \mathbf{q})$ as $\mathrm{CF}(\mathbf{P}, \mathbf{q})=\int d^{3} r^{\prime} S\left(\mathbf{P}, \mathbf{r}^{\prime}\right)\left|\Psi\left(\mathbf{q}^{\prime}, \mathbf{r}^{\prime}\right)\right|^{2}$, with $\mathbf{q}$ being the relative momentum, and where $\Psi$ is the outgoing two-particle wave function, with $\mathbf{q}^{\prime}$ and $\mathbf{r}^{\prime}$ being relative momentum and distance in the pair center-ofmass system. The source function $S$ can be obtained from our simulations, concerning the pair wave function, we follow [5], some details are given in [1].

Here, we investigate $\pi^{+} \pi^{-}$correlations. We calculate the correlation function with Bose-Einstein (BE) quantum statistics included, but no Coulomb corrections. Weak decays are not carried out. We compute correlation functions for different $k_{T}$ interval defined as (in $\mathrm{MeV})$ : $\mathrm{KT} 1=[100,250], \mathrm{KT} 3=[400,550]$, $\mathrm{KT} 5=[700,1000]$, where $k_{T}$ of the pair is defined as $k_{T}=\left(\mid \mathbf{p}_{t}(\right.$ pion 1$)+\mathbf{p}_{t}($ pion 2$\left.) \mid\right) / 2$. In Fig. 2a, we show the results for KT1. We compare the three different scenarios: "full calculation" (solid line), "calculation without hadronic cascade" (dashed), and "calculation without hydro and without cascade" (dotted), and data from ALICE [6]. The data are actually not Coulomb corrected, because the effect is estimated to be small compared to the statistical errors. We consider here the high-multiplicity class, with $d n / d \eta(0)=11.2$, close to the value of 12.9 from our simulated highmultiplicity events. We compare with the real data (not polluted with simulations), normalized via mixed events, and we do the same with our simulations. Despite the limited statistics, in particular at large $k_{T}$, we see very clearly that the "full" scenario, including hydro evolution and hadronic cascade, seems to fit the data much better than the two other ones. Usually people like to extract radii from these distributions, so

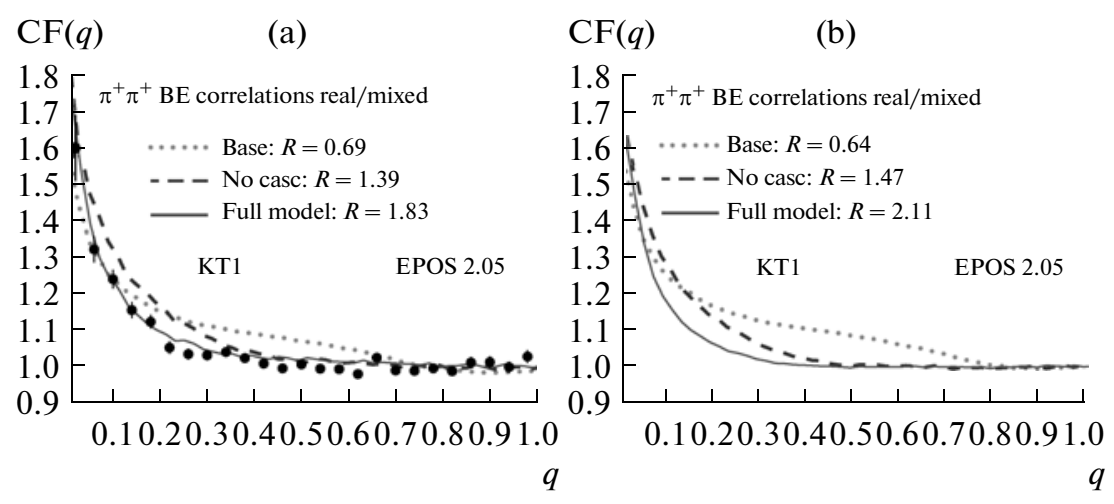

Fig. 2. (Color online) The correlation functions CF for $\pi^{+} \pi^{-}$pairs as obtained from our simulations, for the three different scenarios, for $k_{T}$ bin KT1, compared to data (points). (a) Normalization via mixed events; (b) normalization via a simulation without BE correlation ("bare"). 


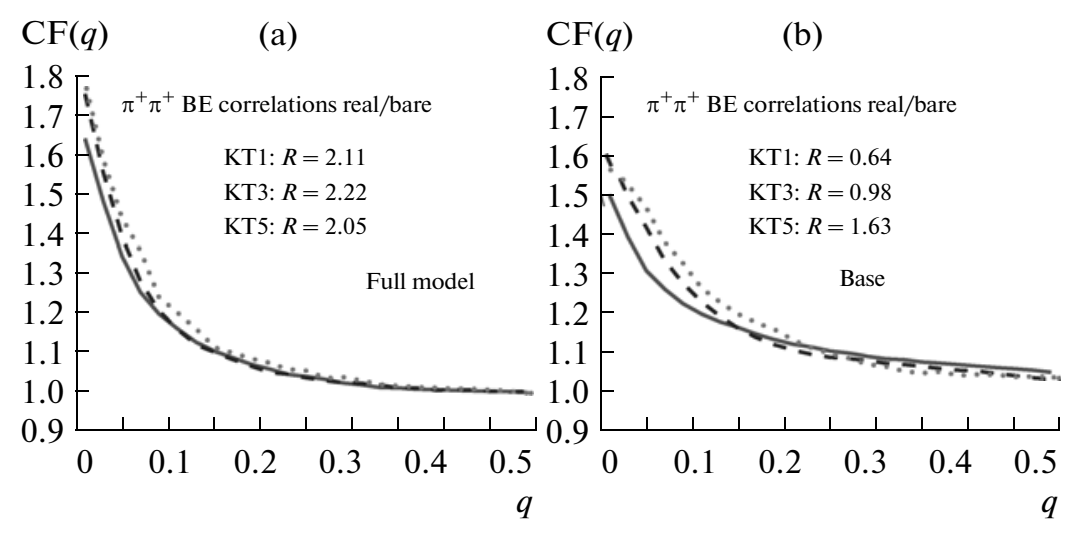

Fig. 3. (Color online) Correlation function, normalized by using a simulation without BE correlation ("bare"), for three $k_{T}$ intervals. (a) Full model; (b) calculation without hydro and without cascade.

when we make a fit of the form $\mathrm{CF}-1=\lambda \exp (-R|\mathbf{q}|)$, in the $|\mathbf{q}|$ range from 0.05 to 0.70 . We obtain the radii given in the figure. So the radii are very different, varying from $0.69 \mathrm{fm}$ (base approach) to $1.80 \mathrm{fm}$ (full model), which is understandable from Fig. 1. We prefer an exponential fit rather than a Gaussian, simply because the former one works, the latter one does not. We do not want to give a precise meaning to $R$, it simply characterizes the distribution.

Normalizing by mixed events is something one can easily do experimentally (this is why we compare with these data), but it is clear that one has still unwanted correlations, like those due to energy-momentum conservation, which is not an issue in mixed events. Doing simulations, life is easier. We can take simulations without Bose-Einstein correlations as base line, rather than mixed events. This is referred to as "real/bare" normalization (to be distinguished from the "real/mixed" case discussed earlier). The corresponding results are shown in Fig. $2 b$, the solid line (full calculation) is now completely horizontal away from the peak region, the radius from the exponential fit is $2.11 \mathrm{fm}$ instead of $1.83 \mathrm{fm}$ for the "mixed" normalization. For the other $k_{T}$ regions, the situation is similar, the final results for all the three $k_{T}$ regions for the full calculation are shown in Fig. 3a, together with the radii from the exponential fit: they are almost identical, around $2 \mathrm{fm}$.

We get to the same conclusion as outlined in [6]: the radii are $k_{T}$-independent, contrary to what has been observed in AuAu scattering. How can it be that our hydrodynamic scenario gives a strong $k_{T}$ dependence in $\mathrm{AuAu}$, but not in $p p$ ? To answer this question, we compute the "true" correlation function (real/bare normalization) for the calculation without hydro and without cascade (just string decay). The results are shown in Fig. 3b. Surprisingly, here we get a strong $k_{T}$ dependence of the radii, but the "wrong" way: we have 0.64 for KT1 and 1.63 fm for KT5! Actually such behavior is quite normal: the distribution is broader for high- $p_{t}$ particles, because high- $p_{t}$ resonances live longer and can move further out before decaying. This effect is in principle also present in AuAu scattering, but it is much more visible for the small $p p$ system. So in $p p$ we have two competing effects:

- radii increase with $k_{T}$, due to the bigger size of the source of the high- $p_{t}$ particles compared to the low- $p_{t}$ ones,

- radii decrease with $k_{T}$, as in AuAu (see [1]), in case of collective flow, due to the $p-x$ correlation.

This $p-x$ correlation exists indeed for the case of hydrodynamic evolutions, and is much smaller in the basic scenario. So in the hydro scenarios, the two competing effects roughly cancel, the radii are $k_{T}$-independent.

To summarize: we employ a hydrodynamic approach to pp scattering at $900 \mathrm{GeV}$. A very interesting application is Bose-Einstein correlations. We have shown that, as in heavy-ion scattering, the hydrodynamic expansion leads to momentum-space correlations, which clearly affect the correlation functions. To see the signal is nontrivial due to the fact that in addition to the $x-p$ correlations (which leads to decreasing radii with $k_{T}$ ), there is a second effect which works the other way round: the single particle source size is $p_{t}$-dependent, which is an important effect in $p p$, not so in heavy-ion scattering. In this sense we can interpret the $k_{T}$ independence of the radii as a real flow effect. Our simulation does not only reproduce the $k_{T}$ independence, but also the whole correlation functions, which is not at all reproduced from the "base scenario" without hydro and without cascade. So the correlation data provide a very strong evidence for a collective hydrodynamic expansion in $p p$ scattering at the LHC.

The research was carried out within the scope of the EUREA: European Ultra Relativistic Energies Agreement (European Research Group: "Heavy Ions 
at Ultrarelativistic Energies”). Iu. K. acknowledges partial support by the National Academy of Sciences of Ukraine (Agreement-2012) and by the State Fund for Fundamental Researches of Ukraine (Agreement2012). T. P. and K. W. acknowledge partial support by a PICS (CNRS) with KIT (Karlsruhe). K. M. acknowledges partial support by the RFBR-CNRS grants no. 08-02-92496-NTsNIL_a and no. 10-0293111-NTsNIL_a.

\section{REFERENCES}

1. K. Werner et al., arXiv:1004.0805; Phys. Rev. C 82, 044904 (2010).
2. K. Werner et al., arXiv:1010.0400; Phys. Rev. C 83, 044915 (2011).

3. M. Bleicher et al., J. Phys. G 25, 1859 (1999); H. Petersen et al., Phys. Rev. C 78, 044901 (2008).

4. G. I. Kopylov and M. I. Podgoretsky, Sov. J. Nucl. Phys. 15, 219 (1972); Sov. J. Nucl. Phys. 18, 336 (1974); S. Pratt, Phys. Rev. Lett. 53, 1219 (1984); M. A. Lisa et al., Ann. Rev. Nucl. Part. Sci. 55, 357-402 (2005); A. Kisiel, W. Florkowski, and W. Broniowski, Phys. Rev. C 73, 064902 (2006).

5. R. Lednicky, Part. Nucl. 40, 307 (2009).

6. ALICE Collab., arXiv:1007.0516. 